\title{
Nondiffracting gravitational waves
}

\author{
Felipe A. Asenjo ${ }^{1, a}$, Sergio A. Hojman ${ }^{2,3,4, b}$ \\ ${ }^{1}$ Facultad de Ingeniería y Ciencias, Universidad Adolfo Ibáñez, Santiago 7491169, Chile \\ 2 Departamento de Ciencias, Facultad de Artes Liberales, Universidad Adolfo Ibáñez, Santiago 7491169, Chile \\ ${ }^{3}$ Departamento de Física, Facultad de Ciencias, Universidad de Chile, Santiago 7800003, Chile \\ ${ }^{4}$ Centro de Recursos Educativos Avanzados, CREA, Santiago 7500018, Chile
}

Received: 20 November 2020 / Accepted: 22 January 2021 / Published online: 30 January 2021

(C) The Author(s) 2021

\begin{abstract}
It is proved that accelerating nondiffracting gravitational Airy wave-packets are solutions of linearized gravity. It is also showed that Airy functions are exact solutions to Einstein equations for non-accelerating nondiffracting gravitational wave-packets.
\end{abstract}

\section{Introduction}

Nondiffracting wave-packets are known solutions to Schrödinger equation [1,2] and Maxwell equations [3,4], and several experimental studies have been dedicated to them [3-11]. These solutions have been observed in experiments and some of their properties have been determined. Some of their features, such as acceleration, are due to the fact that they exhibit non-vanishing Bohm potential [12]. In fact, in Ref. [12] it is established that the usual dispersion relation for massless fields $k_{\mu} k^{\mu}=0$ is valid only when the Bohm potential associated to the solution vanishes, but otherwise the modified right-hand side is exactly equal to the Bohm potential, which may be either positive or negative. It is important to stress that similar results have been found by different authors in the last seventy years [13-35], in addition to the ones already cited above.

Nevertheless, studying the implications of the possible existence of nondiffracting gravitational wave propagation seems to have been neglected so far. To understand its importance on gravitational wave behavior, research in this area is needed. This work intends to help filling that gap.

This manuscript is devoted to investigate accelerating and non-accelerating nondiffracting gravitational waves. We show below how accelerating wave packets are solutions of linearized gravity. Additionally, we explicitly obtain exact non-accelerating, nondiffracting gravitational wave solutions

\footnotetext{
a e-mail: felipe.asenjo@uai.cl (corresponding author)

b e-mail: sergio.hojman@uai.cl
}

that, as far as we know, have not been considered before. This result consists on a new solution for the Ehlers-Kundt waves $[36,37]$.

\section{Accelerating linearized gravitational waves}

First, let us show that accelerating gravitational waves are solutions to linearized gravity. As usual, let us consider a small perturbation $h_{\alpha \beta}$ of a background flat space-time metric $\eta_{\alpha \beta}=(-1,1,1,1)$, such that the total metric is $g_{\alpha \beta}=\eta_{\alpha \beta}+h_{\alpha \beta}$. It is straightforward to show that the tracereversed metric perturbation $\bar{h}_{\alpha \beta}=h_{\alpha \beta}-(1 / 2) \eta_{\alpha \beta} h_{\mu}^{\mu}$ [37], satisfies the free wave equation

$\partial_{\mu} \partial^{\mu} \bar{h}_{\alpha \beta}=0$,

under the Lorenz gauge $\partial_{\beta} \bar{h}_{\alpha}^{\beta}=0$.

In order to exemplify the accelerating solutions for gravitational waves, let us focus in the evolution of the wave polarization $\bar{h}_{z z}=\bar{h}_{z z}(t, x, y)$. Notice that $\partial_{z} \bar{h}_{z z}=0$, thus satisfying the Lorenz gauge. Now we assume the following form for such polarization

$\bar{h}_{z z}(t, x, y)=\mathcal{G}(\zeta, y) \exp (i k \eta)$

where $k$ is an arbitrary constant, $\zeta=x-t$ and $\eta=x+t$ [38]. Thus, as $\bar{h}_{z z}$ evolves according to Eq. (1), we are able to find that $\mathcal{G}$ evolves as

$4 i k \frac{\partial \mathcal{G}}{\partial \zeta}+\frac{\partial^{2} \mathcal{G}}{\partial y^{2}}=0$

This equation is equivalent to a Schrödinger equation for a free particle. It is very well-known that this equation allows solution for accelerating wavepackets [1]. In our case, the 
accelerating gravitational wavepackets that solve Eq. (3) are

$\mathcal{G}(\zeta, y)=\operatorname{Ai}\left(2 k y-k^{2} \zeta^{2}\right) \exp \left(2 i k^{2} \zeta y-\frac{2 i}{3} k^{3} \zeta^{3}\right)$

where Ai is an Airy function. Thus, the nondiffracting accelerating gravitational wavepacket is the real part of full solution (2). This Airy wavepacket has an acceleration equal to $k / 2$, deflecting its trajectory in a parabolic path in the $\zeta-y$ plane [3,39]. Airy waves have been extensively studied in the realm of light propagation in media [40-43]. Beyond the theoretical importance of general accelerating wavepackets, which have encompassed several decades of research, the most remarkable fact is that they have been observed $[9,11,44]$.

Wave solution (4) has infinite energy, which is similar to what happens with plane waves. However, structured nondiffracting gravitational Airy wavepackets with finite energy can be constructed using Gaussian beams $[39,45,46]$. The finite energy gravitational Airy wavepacket is given by

$$
\begin{aligned}
\mathcal{G}(\zeta, y) & \\
= & \operatorname{Ai}\left(2 k y-k^{2} \zeta^{2}+i a k \zeta\right) \\
& \times \exp \left(a k y-a k^{2} \zeta^{2}+\frac{i}{4} a^{2} k \zeta+2 i k^{2} \zeta y-\frac{2 i}{3} k^{3} \zeta^{3}\right),
\end{aligned}
$$

in terms of an arbitrary real parameter $a$ that makes possible the normalization of the solution. The expression given in (5) can be straightforwardly proved to satisfy Eq. (3), and when $a=0$ we recover the wavepacket (4).

We obtain that $\int d y|\mathcal{G}|^{2}=\int d y \mathcal{G}^{*} \mathcal{G}$, is independent of $\zeta$, by using Eq. (3). Then we get that this wavepacket is square integrable along $\zeta=0(x=t)$ as

$$
\int_{-\infty}^{\infty} d y|\mathcal{G}(\zeta, y)|^{2}=\int_{-\infty}^{\infty} d y|\mathcal{G}(0, y)|^{2}=\frac{e^{a^{3} / 12}}{4 k \sqrt{a \pi}}
$$

implying its finite energy.

As it is shown in Ref. [45], the gravitational Airy wavepackets (5) do not accelerate. However, the main maxima lobe of the wave train does show acceleration in the $\zeta-y$ plane. This is exemplified in Fig. 1, where a contour plot in $\zeta-y$ plane of function $|\mathcal{G}(\zeta, y)|=\sqrt{\mathcal{G}^{*}(\zeta, y) \mathcal{G}(\zeta, y)}$ is shown for $a=1$ and $a=2$. In red solid lines we display the evolution of the position of the maxima lobe, that moves in a curved trajectory, thus displaying its acceleration in such plane. (a)

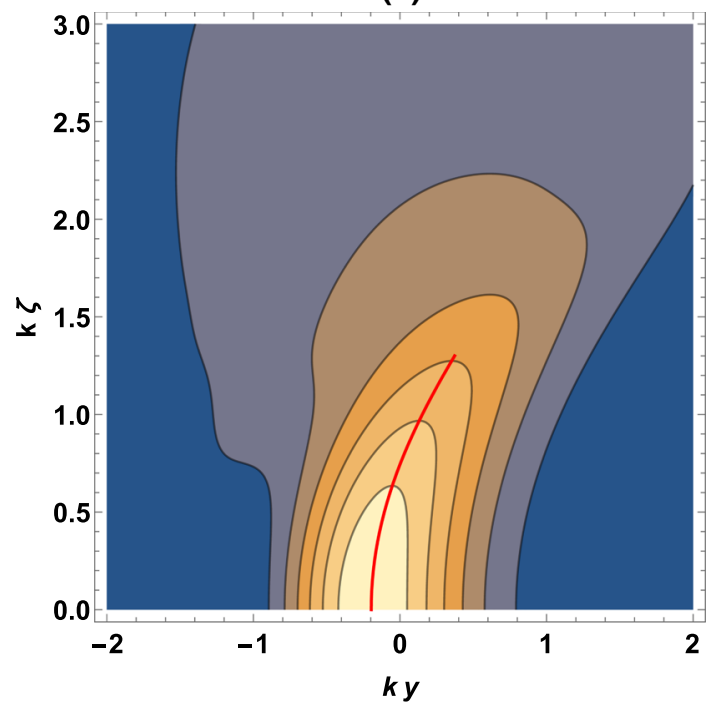

(b)

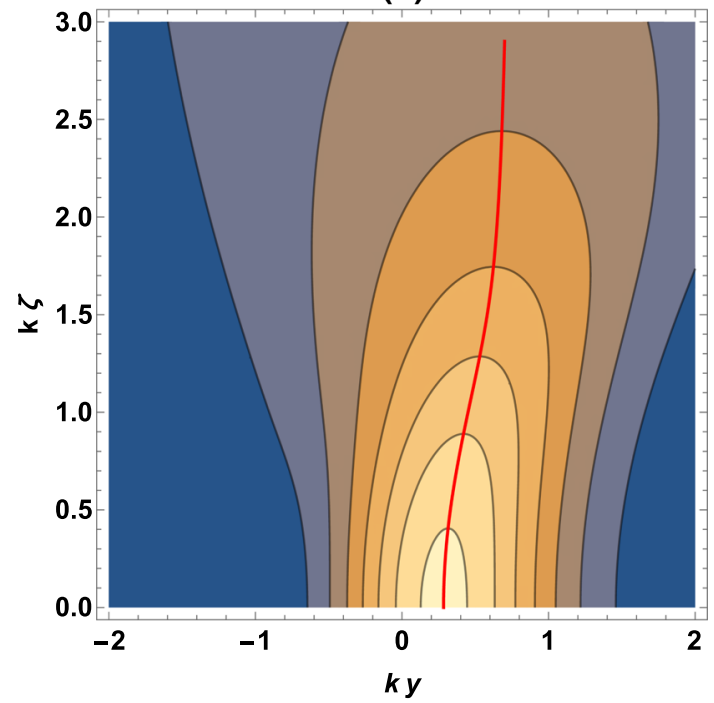

Fig. 1 Contour plot for $|\mathcal{G}(\zeta, y)|$, with $\mathcal{G}$ given in Eq. (5), in terms of normalized $k \zeta$ and $k y$ variables, for different values of $a$. a Plot for $a=1$. b Plot $a=2$. For both cases the red solid line shows the dynamical trajectory of the position of local maxima lobes of the wavepacket, which shows acceleration in the $\zeta-y$ plane. The colors get brighter as the value of $|\mathcal{G}(\zeta, y)|$ increases

\section{Exact nondiffracting gravitational waves}

Structured wave-packets are not only solutions to the linearized Einstein equations, but they are also exact solutions to the full Einstein equations. Exact nondiffracting gravitational waves can be obtained from a modification of EhlersKundt solution [36,37]. Consider the exact metric in cartesian coordinates given by the interval

$d s^{2}=-d t^{2}+L(u)^{2} d x^{2}+W(u)^{2} d y^{2}+d z^{2}$, 
where $L$ and $W$ are arbitrary functions of $u=z-t$. Einstein equations are satisfied when

$\frac{1}{L} \frac{d^{2} L}{d u^{2}}+\frac{1}{W} \frac{d^{2} W}{d u^{2}}=0$.

These waves, described by metric (7), are $p p$-waves [37,47]. This can be seen by applying the coordinate transformation $x=X / L, y=Y / W, v=V-X^{2} d_{u} L / L-Y^{2} d_{u} W / W$, together with Eq. (8), to put the metric (7) in the Kerr-Schild form $d s^{2}=d X^{2}+d Y^{2}-d u d V+d u^{2}\left(X^{2}-Y^{2}\right) d_{u}^{2} L / L$. For these classes of metrics linear and non-linear theories coincide under certain conditions [48].

Here we show that there are certain solutions of Eq. (8) that describe exact nondiffracting and non-accelerating gravitational waves. The simplest non-trivial solution for a structured gravitational wave can be obtained in terms of Airy functions

$$
\begin{gathered}
L(u)=L_{0} \operatorname{Ai}(u), \\
W(u)=W_{0} \operatorname{Ai}(-u),
\end{gathered}
$$

with arbitrary constants $L_{0}$ and $W_{0}$. These nondiffracting wave-packets have the property that the intensity of the gravitational wave is concentrated in the caustic. However, these exact Airy gravitational waves have infinite energy. This is a feature that they share with other gravitational waves, such plane waves [37]. Nevertheless, what is relevant in this exact solution is its structured configuration.

\section{Discussion}

Nondiffracting wave-packets have been a fruitful field of study in quantum mechanics $[1,2,49,50]$, electromagnetism and optics [3-11]. The solutions (4) and (9) presented along this work are in the same spirit, and complement others solutions for structured gravitational beams, as Bessel or Laguerre-Gauss, found in linearized general relativity $[51,52]$.

Although simple in their mathematical form, the new linearized accelerating and exact non-accelerating solutions can bring new insights in the form that different gravitational waves propagate in a flat space-time background.

How the nondiffracting features are manifested for gravitational waves in curved space-time is an open question currently under investigation.

Data Availability Statement This manuscript has no associated data or the data will not be deposited. [Authors' comment: This is a theoretical study and no experimental data has been listed.]

Open Access This article is licensed under a Creative Commons Attribution 4.0 International License, which permits use, sharing, adaptation, distribution and reproduction in any medium or format, as long as you give appropriate credit to the original author(s) and the source, pro- vide a link to the Creative Commons licence, and indicate if changes were made. The images or other third party material in this article are included in the article's Creative Commons licence, unless indicated otherwise in a credit line to the material. If material is not included in the article's Creative Commons licence and your intended use is not permitted by statutory regulation or exceeds the permitted use, you will need to obtain permission directly from the copyright holder. To view a copy of this licence, visit http://creativecomm ons.org/licenses/by/4.0/.

Funded by SCOAP 3 .

\section{References}

1. M.V. Berry, N.L. Balazs, Am. J. Phys. 47, 264 (1979)

2. C. Yuce, Phys. Lett. A 380, 3791 (2016)

3. I. Kaminer, R. Bekenstein, J. Nemirovsky, M. Segev, Phys. Rev. Lett. 108, 163901 (2012)

4. S. Hacyan, J. Opt. 13, 105710 (2011)

5. P. Aleahmad, H. Moya Cessa, I. Kaminer, M. Segev, D.N. Christodoulides, J. Opt. Soc. Am. A 33, 2047 (2016)

6. I.D. Chremmos, N.K. Efremidis, Phys. Rev. A 88, 063816 (2013)

7. N.K. Efremidis, Z. Chen, M. Segev, D.N. Christodoulides, Optica 6, 686 (2019)

8. I. Kaminer, J. Nemirovsky, M. Segev, Opt. Exp. 20, 18827 (2012)

9. A. Patsyk, M.A. Bandres, R. Bekenstein, M. Segev, Phys. Rev. X 8, 011001 (2018)

10. M.A. Bandres, J.C. Gutiérrez-Vega, Opt. Exp. 15, 16719 (2007)

11. H. Esat Kondakci, A.F. Abouraddy, Phys. Rev. Lett. 120, 163901 (2018)

12. S.A. Hojman, F.A. Asenjo, Phys. Scr. 95, 085001 (2020)

13. J. Slepian, Elec. Eng. 68, 1080 (1949)

14. G.V. Skrotskii, Soviet Phys. Doklady 1957(2), 226 (1957)

15. J. Plebanksi, Phys. Rev. 118, 1396 (1960)

16. B.S. DeWitt, R.W. Brehme, Ann. Phys. (N.Y.) 9, 220 (1960)

17. G. Velo, D. Zwanziger, Phys. Rev. 186, 1337 (1969)

18. G. Velo, D. Zwanziger, Phys. Rev. 188, 2218 (1969)

19. B. Mashhoon, Phys. Rev. D 8, 4297 (1973)

20. A.J. Hanson, T. Regge, Ann. Phys. 87, 498 (1974)

21. B. Mashhoon, Phys. Rev. D 11, 2679 (1975)

22. B. Mashhoon, Ann. Phys. 89, 254 (1975)

23. S.A. Hojman, Electromagnetic and gravitational interactions of a spherical relativistic top. Ph.D. thesis, Princeton University (1975) (unpublished)

24. S. Hojman, T. Regge, Studies in Mathematical Physics, Essays in Honor of Valentin Bargmann, Ed. E.H. Lieb, B. Simon, A.S. Wightman, Princeton University Press, pg. 195 (1976)

25. S. Hojman, Phys. Rev. D 18, 2741 (1978)

26. R. Bekenstein, J. Nemirovsky, I. Kaminer, M. Segev, Phys. Rev. X 4, 011038 (2014)

27. F.A. Asenjo, S.A. Hojman, Eur. Phys. J. C 77, 732 (2017). https:// doi.org/10.1140/epjc/s10052-017-5330-7

28. F.A. Asenjo, S.A. Hojman, Class. Quantum Grav. 34, 205011 (2017)

29. F.A. Asenjo, S.A. Hojman, Phys. Rev. D 96, 044021 (2017)

30. N.I. Petrov, Sci. Rep. 9, 18332 (2019). https://doi.org/10.1038/ s41598-019-54921-5

31. D. Mugnai, A. Ranfagni, R. Ruggeri, Phys. Rev. Lett 84, 4830 (2000)

32. D. Giovannini, J. Romero, V. Potoček, G. Ferenczi, F. Speirits, S.M. Barnett, D. Faccio, M.J. Padgett, Sciencexpress 3035, 10.1126 (2015)

33. F. Bouchard, J. Harris, H. Mand, R.W. Boyd, E. Karimi, Opt. Lett. 3, 351 (2016) 
34. R. Bekenstein, Y. Kabessa, Y. Sharabi, O. Tal, N. Engheta, G. Eisenstein, A.J. Agranat, M. Segev, Nature Photon. 11, 664 (2017)

35. H.E. Kondakci, A.F. Abouraddy, Nat. Commun. 10, 929 (2019)

36. J. Ehlers, W. Kundt, Gravitation: an introduction to current research, ed. L. Witten, Wiley, New York (1962)

37. C.W. Misner, K.S. Thorne, J.A. Wheeler, Gravitation (W. H. Freeman and Co., New York, 1973)

38. I.M. Besieris, A.M. Shaarawi, R.W. Ziolkowski, Am. J. Phys. 62, 519 (1994)

39. G.A. Siviloglou, D.N. Christodoulides, Opt. Lett. 32, 979 (2007)

40. C. Chen et al., J. Opt. 17, 035504 (2015)

41. B. Chen et al., J. Opt. 18, 055504 (2016)

42. C. Chen et al., J. Opt. 18, 055505 (2016)

43. Y. Peng et al., Opt. Commun. 359, 116 (2016)

44. G.A. Siviloglou, J. Broky, A. Dogariu, D.N. Christodoulides, Phys. Rev. Lett. 99, 213901 (2007)
45. J. Lekner, Eur. J. Phys. 30, L43 (2009)

46. I.M. Besieris, A.M. Shaarawi, Opt. Lett. 32, 2447 (2007)

47. J.B. Griffiths, Collinding Plane Waves in General Relativity (Dover Publications Inc., New York, 1991)

48. E. Kilicarslan, Phys. Rev. D 99, 124048 (2019)

49. I. Kaminer, J. Nemirovsky, M. Rechtsman, R. Bekenstein, M. Segev, Nature Phys. 11, 261 (2015)

50. N. Voloch-Bloch, Y. Lereah, Y. Lilach, A. Gover, A. Ari, Nature 494, 331 (2013)

51. I. Bialynicki-Birula, Z. Bialynicka-Birula, New J. Phys. 18, 023022 (2016)

52. A. Paták, M. Zouhar, T. Řiháček, O. Brzobohatý, J. Geršl, A. Charvátová Campbell, Phys. Rev. D 100, 044050 (2019) 